\title{
Influence of Source Interference on the Directivity of Jet Mixing Noise
}

\author{
Ulf Michel * \\ DLR, German Aerospace Center, Berlin, 10623 Germany
}

\begin{abstract}
A simple model is proposed for the description of the interference effects on the radiation of jet mixing noise. It is used to show analytically that many experimental observations in the acoustic far field of a jet can be explained with the acoustic analogy. This relates not only to the directivity of a static jet as was already shown by Lighthill, but also to the unexpectedly high noise radiation into the forward arc in flight. It also explains the experimental evidence that the peak frequency of a static jet is almost constant over a wide range of emission angles for a static jet and is subject to a Doppler frequency shift in flight. It is necessary to describe the turbulent flow quantities in the coordinate system fixed on the nozzle, in which the source quantities are stationary random and the limits of the source integral are stationary. An integral for the power-spectral density is derived, which includes the quadrupole and the dipole sources. Turbulence convection is considered through a phase angle of the cross-spectral density of the sources. The influence of source interference is expressed in terms of an interference integral which describes the sound radiation of one source volume element. In order to achieve analytic solutions for this integral, the radial extension of the jet is neglected and simple models are introduced for the decay of the coherence with increasing axial separation of the source positions. The decay is shown to have a large influence on the radiation, especially into the forward arc.
\end{abstract}

\section{Nomenclature}

$\begin{array}{lll}a_{0} & \text { sound speed in the ambience of the jet } & \\ D_{f} & \text { Doppler factor } & W_{q q c} \\ D_{j} & \text { nozzle diameter } & \\ f & \text { frequency } & W_{q q s} \\ F_{d} & \text { interference integral for dipole sources } & \\ F_{q} & \text { interference integral for quadrupole sources } & W_{q q} \\ M a_{f} & \text { flight Mach number } & W_{d d} \\ p, p^{\prime} & \text { pressure fluctuation } & x_{i} \\ p_{0} & \text { atmospheric pressure in ambience } & y_{i} \\ q & \text { source term } & \gamma_{d} \\ q_{i j} & \text { quadrupole source quantity } & \\ q_{i} & \text { dipole source quantity } & \gamma_{q} \\ Q_{q} & \text { quadrupole source term } & \\ & \text { in acoustic far field } & \theta \\ Q_{d} & \text { dipole source term } & \theta_{e} \\ & \text { in acoustic far field } & \eta_{i} \\ r & \text { geometric distance between } & \\ & \text { source and observer } & \psi_{q} \\ r_{e} & \text { emission (or wave-normal) distance } & \\ & \text { between source and observer } & \psi_{d} \\ t & \text { time } & \\ t_{r} & \text { retarded time } & \psi_{r} \\ U_{f} & \text { flight speed } & \\ U_{i} & \text { velocity vector of flight stream } & \rho \\ U_{j} & \text { jet speed } & \rho_{0} \\ U_{j} / a_{0} & \text { acoustic Mach number of jet } & \sigma \\ V & \text { integration volume of source region } & {[\ldots]} \\ V_{c} & \text { integration volume of coherence region } & \\ W_{p p} & \text { power-spectral density of pressure } & \\ & \text { pow } & \end{array}$

\author{
fluctuations \\ power-spectral density of source term $Q_{q}$ \\ in source position $y_{i}+\eta_{i}$ \\ power-spectral density of source term $Q_{q}$ \\ in source position $y_{i}$ \\ cross-spectral density of source term $Q_{q}$ \\ cross-spectral density of source term $Q_{d}$ \\ observer position \\ source position \\ coherence of $Q_{d}$ between two \\ source positions \\ coherence of $Q_{q}$ between two \\ source positions \\ angle between source and observer \\ emission (wave normal) angle \\ separation vector between two \\ source positions \\ phase difference due to source motion \\ of quadrupole sources \\ phase difference due to source motion \\ of dipole sources \\ phase difference due to retarded time \\ difference \\ density \\ density of ambient fluid \\ jet stretching factor due to flight speed \\ term evaluated at the retarded time
}

* Senior Scientist, DLR, German Aerospace Center, Institute of Propulsion Technology, Engine Acoustics Branch, Müller-Breslau-Str. 8, 10623 Berlin, Germany, Honorary Professor Aeroacoustics, BTU Cottbus, Germany, ulf.michel@dlr.de, Member AIAA and DGLR 


\section{Introduction}

$\mathrm{T}$ HE sound generated by a free jet when it mixes with the ambience is an important contribution to the total noise emission of jet powered transport aircraft and it is the dominating noise source of military combat aircraft. The theory for the underlying sound generation process was developed by Lighthill. ${ }^{1,2}$ Ffowcs Williams ${ }^{3}$ included the influence of a flight speed. Lighthill and Ffowcs Williams discussed the sound radiation problem in a coordinate system fixed on the moving frame of turbulent "eddies" which were considered to be the sources of sound. With assumptions on how the sources scale on mean flow quantities Lighthill concluded that the sound intensity of a jet at 90 degrees to the axis is proportional to eighth power of the acoustic Mach number $U_{j} / a_{0}$, where $U_{j}$ is the jet speed and $a_{0}$ the speed of sound in the ambience of the flow. The theory explained why the sound pressure level in the far field of a jet is larger in the rear arc (in the direction of the jet's mean velocity vector) than in the forward arc.

However, other experimental findings could not be explained with these results, e.g., the deviations from the eighth power relation for low jet speeds and for hot jets, the additional noise generated by supersonic jets, and the unexpectedly high noise radiation into the forward arc when the aircraft is in flight. In addition, the frequencies found experimentally in the rear arc are not higher than in the forward arc as was expected from the assumption of moving sources (they are even lower) and the frequencies in flight are modified according to the Doppler frequency shift with respect to the motion of the nozzle. Both findings suggest that the sources have to be described in the coordinate system fixed on the nozzle.

Ribner ${ }^{4}$ was first to use such a coordinate system in which the turbulence satisfies the mathematically important condition of stationary randomness. The motion of the sources was considered by the cross-correlation function of the source quantities. This approach was later applied by Michalke ${ }^{5}$ in the frequency domain when he introduced a wave model to describe the turbulence in the jet. Michalke expanded the source region into azimuthal components ${ }^{6}$ and discussed the influence of source coherence on the sound radiation. ${ }^{7,8}$ This means that the sound radiation of instability waves with growing and decaying amplitudes was already considered in the 1970s, a work that is unfortunately not properly cited in most of the papers dealing with the sound radiation of jets. Michalke's approach was later extended by Michalke \& Michel ${ }^{9,10}$ to include the effects of flight speed and of density non-uniformities. Frequency spectra were discussed by Michel \& Michalke. ${ }^{11,12}$ It was shown by Michel ${ }^{13}$ that broadband shock noise can also be described with this variant of the acoustic analogy. Broadband shock noise is the result of a special form of Mach wave radiation.

The nozzle-fixed coordinate system was also used by Tam \& Auriault ${ }^{14}$ in their semi-empirical theory for the prediction of jet mixing noise. This choice was one condition for their success in predicting the spectral shape of the far-field noise as function of emission angle.

This paper is written with the objective of providing a source model for the acoustic analogy in the coordinate system of the nozzle, which is used in experiments and numerical simulations. The source model includes the effects of source coherence. A proper modeling of the coherence and interference of the distributed source field of a jet is likely important, when the noise emission of aeroengines is analyzed with microphone arrays in the geometric near field of the engine. The cross-spectral density matrix of the microphones is influenced by the coherence in the source field of the jet and it is very likely that the source distribution cannot be determined correctly with a point source model that neglects the source coherence. Therefore, the development of a model for the source coherence and interference inside the jet flow appears to be very important.

In order to include the effects of flight speed, the convective Lighthill equation is used for the analysis. The derivation of the solution for the power-spectral density in the geometric far field is presented in detail, although it was previously presented by Michel $\&$ Michalke, ${ }^{12}$ a publication that is not readily available.

\section{Sound generation by free turbulence}

\section{A. Convective Lighthill equation}

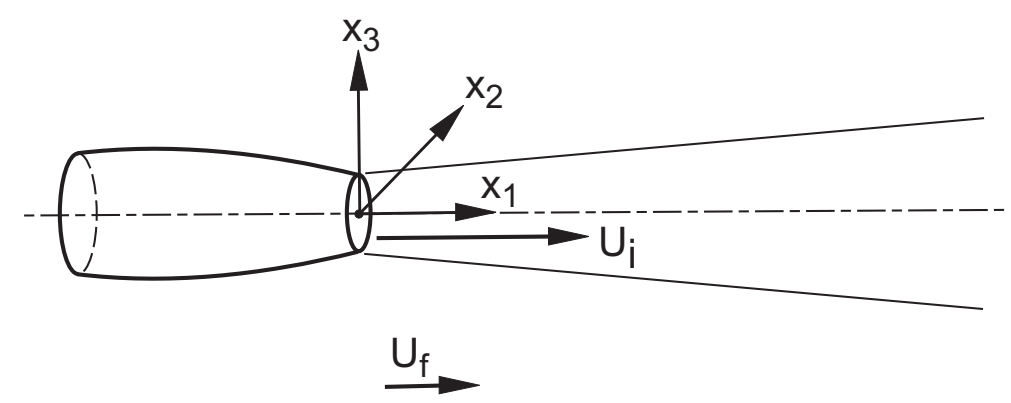

Figure 1. Turbulent jet with jet speed $U_{j}$ as source of sound surrounded by a fluid with uniform flow speed $U_{f}$. 
The situation of a jet in forward motion is shown in figure 1 in a coordinate system fixed on the nozzle. This coordinate system requires the use of the convective form of the Lighthill equation, which is given for the pressure $p$ by

$$
\frac{1}{a_{0}^{2}}\left(\frac{\partial}{\partial t}+U_{i} \frac{\partial}{\partial x_{i}}\right)^{2} p-\frac{\partial^{2} p}{\partial x_{i}^{2}}=q .
$$

$x_{i}$ describes the position relative to the nozzle center, $U_{i}$ is the uniform flow velocity vector of the surrounding flow, and $a_{0}$ is the sound speed in the ambient flow. The source term $q$ on the right hand side of equation (1) is given by (see Michalke \& $\operatorname{Michel}^{9}$ )

$$
q=\frac{\partial^{2}}{\partial x_{i} \partial x_{j}}\left(\rho u_{i} u_{j}-\tau_{i j}\right)-\left(\frac{\partial}{\partial t}+U_{i} \frac{\partial}{\partial x_{i}}\right)^{2}\left(\rho-\frac{p}{a_{0}^{2}}\right),
$$

where $u_{i}=c_{i}-U_{i}$ is the difference between the local velocity $c_{i}$ and the constant velocity $U_{i}$ in the ambience. $q$ is quadratically small outside the turbulent flow region. Equation (1) with $q=0$ describes the sound propagation in this space.

Eqs. (1) and (2) are exact because they are derived from the Navier-Stokes equations without restricting assumptions. If we assume that the entropy remains constant along streaklines, which means that the viscous dissipation and heat conduction have negligible influences on the sound generation, equation (2) can be approximated by

$$
q=\frac{\partial^{2} q_{i j}}{\partial x_{i} \partial x_{j}}+\frac{\partial q_{i}}{\partial x_{i}}
$$

where the two terms $q_{i j}$ and $q_{i}$ are defined by

$$
q_{i j}=\rho_{o} u_{i} u_{j}\left(1+\frac{p^{\prime}}{\rho_{o} a_{0}^{2}}\right)-\left(1-\frac{\rho_{o}}{\rho}\right) p^{\prime} \delta_{i j},
$$

and

$$
q_{i}=p^{\prime} \frac{\partial}{\partial x_{i}}\left(\frac{\rho_{o}}{\rho}\right)
$$

$p^{\prime}=p-p_{0}$ is the difference between the local pressure $p$ inside the source region and the pressure $p_{0}$ in the ambience. $a_{0}$ is the speed of sound in the ambience. Terms of order $p^{\prime 2} /\left(\rho_{0} a_{0}^{2}\right)$ are neglected.

The first term on the right hand side of equation (3) describes a quadrupole source distribution because of the second spatial derivative of the tensor $q_{i j}$. This term yields the well known $\left(U_{j} / a_{0}\right)^{8}$ intensity relation of Lighthill ${ }^{1,2}$ for the noise emission of a turbulent jet in a direction normal to the jet axis.

The second term on the right hand side of equation (3), the unsteady density source term, describes a dipole source distribution because of the first spatial derivative. The unsteady density source terms were discussed by Ffowcs Williams ${ }^{15}$ but it was first shown by Morfey ${ }^{16}$ that these terms may be important for hot jets. The unsteady density source term leads to a $\left(U_{j} / a_{0}\right)^{6}$ intensity relation (Michalke \& Michel $^{10}$ ) normal to the jet axis.

\section{B. Solution of the convective Lighthill equation}

\section{Emission coordinates}

The solution of the convective Lighthill equation (1) for the case of radiation into free space can be expressed in a very compact form as function of the emission (or wave-normal) angle $\theta_{e}$ and the emission distance $r_{e}$, which are defined in figure 2. $\theta_{e}=0$ is oriented in the flight direction. $r_{e}$ is the wave-normal component of the distance between the source element $\mathrm{d} V\left(y_{i}\right)$ and the observer position. While the sound waves propagate from the source position to the observer, they are convected by a distance of $M a_{f} r_{e}$ in the downstream direction, where $M a_{f}=U_{f} / a_{0}$ is the flight Mach number.

The emission distance $r_{e}$ and the emission angle $\theta_{e}$ can be computed from the geometric distance $r$ and the observer angle $\theta$ as follows.

$$
r_{e}=\frac{r}{\sqrt{1-M a_{f}^{2} \sin ^{2} \theta}-M a_{f} \cos \theta}
$$

and

$$
\cos \theta_{e}=\cos \theta\left[\sqrt{1-M a_{f}^{2} \sin ^{2} \theta}-M a_{f} \cos \theta\right]+M a_{f} .
$$

The inverse relations are

$$
r=r_{e} \sqrt{1-2 M a_{f} \cos \theta_{e}+M a_{f}^{2}}
$$




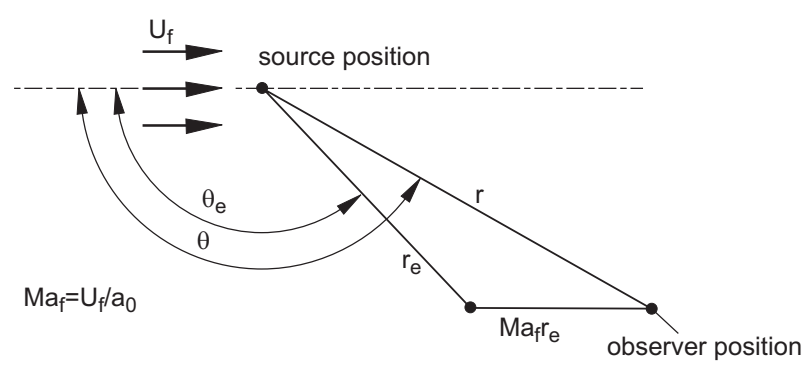

Figure 2. Relation between the source position and the observer position in a uniform flow (wind tunnel coordinate system).

and

$$
\cos \theta=\frac{\cos \theta_{e}-M a_{f}}{\sqrt{1-2 M a_{f} \cos \theta_{e}+M a_{f}^{2}}} .
$$

\section{Integral solution}

The solution for the sound pressure $p^{\prime}=p-p_{0}$ in an observer position $x_{i}$ is then given by the Kirchhoff integral (see Michalke \& Michel $^{10}$ ).

$$
p^{\prime}\left(x_{i}, t\right)=\frac{1}{4 \pi} \int_{V} \frac{1}{r_{e} D_{f}}\left[\frac{\partial^{2} q_{i j}}{\partial x_{i} \partial x_{j}}\right] \mathrm{d} V\left(y_{i}\right)+\frac{1}{4 \pi} \int_{V} \frac{1}{r_{e} D_{f}}\left[\frac{\partial q_{i}}{\partial x_{i}}\right] \mathrm{d} V\left(y_{i}\right) .
$$

The sound pressure is described by two integrals over the whole volume $V$ that is occupied by the turbulent flow. The brackets in equation (10) indicate that the integrands have to be evaluated at the retarded time

$$
t_{r}=t-r_{e} / a_{0}
$$

$D_{f}$ is the Doppler factor, which is defined by

$$
D_{f}=1-M a_{f} \cos \theta_{e}
$$

equation (10) is valid everywhere in an unbounded space, even inside the turbulent flow.

\section{Acoustic far field}

The two source terms in equation (10) simplify in the acoustic far field of the sources, where the spatial derivatives can be replaced by time derivatives. This is shown by Michalke $\&$ Michel $^{10}$ for the convective wave equation.

$$
\begin{aligned}
\frac{\partial^{2} q_{i j}}{\partial x_{i} \partial x_{j}} & =\frac{1}{a_{0}^{2} D_{f}^{2}} \frac{\partial^{2} q_{q}}{\partial t^{2}} \\
\frac{\partial q_{i}}{\partial x_{i}} & =\frac{1}{a_{0} D_{f}} \frac{\partial q_{d}}{\partial t}
\end{aligned}
$$

The influence of the Doppler factor $D_{f}$ increases the source intensity against the flow direction $\left(\theta_{e}<90^{\circ}\right)$, the increase being larger for the quadrupole sources $q_{q}$ than for the dipole sources $q_{d}$.

The new source quantities $q_{q}$ and $q_{d}$ are defined by (Michalke \& Michel ${ }^{10}$ )

$$
\begin{gathered}
q_{q}\left(y_{i}, \theta_{e}, t\right)=\rho_{0} u_{r_{e}}^{2}\left(1+\frac{p^{\prime}}{\rho_{0} a_{0}^{2}}\right)-\left(1-\frac{\rho_{0}}{\rho}\right) p^{\prime}, \\
q_{d}\left(y_{i}, \theta_{e}, t\right)=p^{\prime} \frac{\partial}{\partial y_{r_{e}}}\left(\frac{\rho_{0}}{\rho}\right) .
\end{gathered}
$$

For small values $p^{\prime}$ within the source region and for $\rho \approx \rho_{0}$ equation (15) can be approximated by

$$
q_{q} \approx \rho_{0} u_{r e}^{2} .
$$


The quadrupole source strength is dominated by the second time derivative of the square of the velocity fluctuations in the volume element $\mathrm{d} V\left(y_{i}\right)$ in the direction $\theta_{e}$ toward the observer position $x_{i}$.

The dipole source strength is determined by the product of the first time derivative of the local pressure fluctuations $p^{\prime}$ und the derivative of the inverse of the density gradient in the direction $\theta_{e}$ toward the observer position $x_{i}$.

The solution for the sound pressure $p^{\prime}=p-p_{0}$ in an observer position $x_{i}$ in the acoustic far field and in free space is then given by (see Michalke \& Michel $^{10}$ ).

$$
p^{\prime}\left(x_{i}, t\right)=\frac{1}{4 \pi a_{0}^{2}} \int_{V} \frac{\left[Q_{q}\right]}{r_{e} D_{f}^{3}} \mathrm{~d} V\left(y_{i}\right)+\frac{1}{4 \pi a_{0}} \int_{V} \frac{\left[Q_{d}\right]}{r_{e} D_{f}^{2}} \mathrm{~d} V\left(y_{i}\right) .
$$

The quadrupole source term $Q_{q}$ and the dipole source term $Q_{d}$ in equation (18) are abbreviations for

$$
\begin{aligned}
Q_{q}\left(y_{i}, \theta_{e}, t\right) & =\frac{\partial^{2} q_{q}\left(y_{i}, \theta_{e}, t\right)}{\partial t^{2}} \\
Q_{d}\left(y_{i}, \theta_{e}, t\right) & =\frac{\partial q_{d}\left(y_{i}, \theta_{e}, t\right)}{\partial t}
\end{aligned}
$$

\section{Time averaged solutions}

\section{Power-spectral density}

One is generally not interested in the time-dependent solution for the sound pressure (like equation (18)) but in time averaged quantities. The power-spectral density function is studied here because it is required for the description of the important interference effects in the geometric far field of distributed sources.

The power-spectral density $W_{p p}\left(x_{i}, f\right)$ of the pressure fluctuations in the observer position $x_{i}$ as function of the frequency $f$ in the coordinate system of the nozzle is given by

$$
W_{p p}\left(x_{i}, f\right)=W_{p p q q}\left(x_{i}, f\right)+W_{p p d d}\left(x_{i}, f\right) .
$$

It has contributions from the two source terms $Q_{q}$ and $Q_{d}$, which are assumed to be uncorrelated.

\section{Quadrupole noise}

The power-spectral density $W_{p p q q}\left(x_{i}, f\right)$ of the pressure fluctuations due to the quadrupole source term $Q_{q}$ in the acoustic far field is given by the double integral

$$
W_{p p q q}\left(x_{i}, f\right)=\frac{1}{\left(4 \pi a_{0}^{2}\right)^{2}} \int_{V} \frac{1}{r_{e} D_{f}^{3}} \int_{V_{c}} \frac{1}{r_{e c} D_{f c}^{3}} \operatorname{Re}\left\{W_{q q}\left(y_{i}, y_{c i}, f\right) \exp \left(i \psi_{r}\right)\right\} \mathrm{d} V_{c}\left(y_{c i}\right) \mathrm{d} V\left(y_{i}\right)
$$

with the cross-spectral density $W_{q q}$ of the source term $Q_{q}$ between the two positions $y_{i}$ and $y_{c i}=y_{i}+\eta_{i}$, where $\eta_{i}$ is the separation vector between the two volume elements.

$$
W_{q q}\left(y_{i}, \eta_{i}, f\right)=\int_{-\infty}^{\infty} \overline{Q_{q}\left(y_{i}, t\right) Q_{q}\left(y_{i}+\eta_{i}, t+\tau\right)} \exp (i 2 \pi f \tau) \mathrm{d} \tau
$$

$Q_{q}$ is defined by equation (19). $R e\{\ldots\}$ means real part of. $r_{e}$ and $D_{f}$ are the emission (wave-normal) distance and Doppler factor of the volume element $\mathrm{d} V\left(y_{i}\right)$, respectively, and $r_{e c}$ and $D_{f c}$ are the corresponding values for the volume element $\mathrm{d} V_{c}\left(y_{i}\right)$.

The influence of the emission distance $r_{e}$ on the retarded times is considered in equation (22) through the phase difference $\psi_{r}$, which is given by

$$
\psi_{r}=2 \pi f \frac{r_{e}-r_{e c}}{a_{0}}=k\left(r_{e}-r_{e c}\right)
$$

where $k=2 \pi f / a_{0}$ is the wave number.

The cross-spectral density $W_{q q}$ depends on the source positions $y_{i}$ and $y_{c i}=y_{i}+\eta_{i}$ of the two volume elements $\mathrm{d} V_{c}\left(y_{c i}\right)$ and $\mathrm{d} V\left(y_{i}\right)$, on the frequency $f$, the emission angles $\theta_{e}$ and $\theta_{e c}$ in the two source positions and all other parameters that influence the turbulence in the flow. Since the cross-spectral density is a complex quantity it can be expressed in terms of its amplitude and phase as follows

$$
\begin{aligned}
W_{q q} & =\left|W_{q q}\left(y_{i}, y_{c i}\right)\right| \exp \left(i \psi_{q}\right) \\
& =\sqrt{W_{q q}\left(y_{i}, y_{i}\right) W_{q q}\left(y_{c i}, y_{c i}\right)} \gamma_{q}\left(y_{i}, y_{c i}\right) \exp \left(i \psi_{q}\right)
\end{aligned}
$$


The phase difference $\psi_{q}$ is related to the phase speed of the disturbances in the jet's turbulence, which in turn is the result of turbulence convection. $\gamma_{q}$ is the coherence of the source terms $Q_{q}$ between the two source positions $y_{i}$ and $y_{c i}$.

Eqs. (22), (24), and (26) are valid in the acoustic far field of the sources, but the observer position may be located in the geometric near field of the jet.

Equation (22) may be abbreviated as follows

$$
W_{p p q q}\left(x_{i}, f\right)=\frac{1}{\left(4 \pi a_{0}^{2}\right)^{2}} \underbrace{\int_{V}^{V} \frac{W_{q q s}}{r_{e}^{2} D_{f}^{6}} F_{q} \mathrm{~d} V\left(y_{i}\right)}_{\text {integral over source volume }} .
$$

with the abbreviation for the inner integral

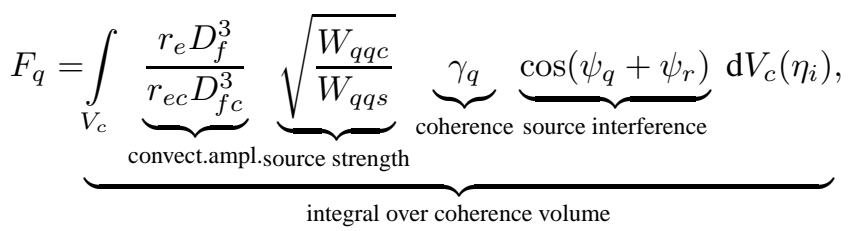

$W_{q q s}=W_{q q}\left(y_{i}, y_{i}, f, \theta_{e}\right)$ is the power-spectral density of $Q_{q}$ in the source position $y_{i}$ and $W_{q q c}=$ $W_{q q}\left(y_{c i}, y_{c i}, f, \theta_{e c}\right)$ is the corresponding value in the second position $y_{c i}=y_{i}+\eta_{i}$. Both values describe the strength of the source term $Q_{q}$ as function of the frequency $f$ and emission angle $\theta_{e}$ in the two source positions. $W_{q q c} / W_{q q s}$ is the relative source strength, where the source strength in position $y_{i}+\eta_{i}$ is normalized with the strength in position $y_{i} . F_{q}$ is a normalized contribution of one source element $\mathrm{d} V\left(y_{i}\right)$ to the far field of the jet. It describes the interference effects between the power-spectral density $W_{q q s}$ in the source element $\mathrm{d} V\left(y_{i}\right)$ and its complete neighborhood. $\gamma_{q}$ indicates that only the coherent part of the fluctuations in the two positions contributes to the sound in the far field. The integration over the coordinate $\eta_{i}=y_{c i}-y_{i}$ in equation (28) needs only to be carried out over the region in which the coherence $\gamma_{q}$ is not negligible. This region is termed coherence volume in the corresponding under-brace of equation (28).

The source interference term in equation (28) describes the phase relationship between the contributions from different source positions. The phase $\psi_{q}$ considers the effect of source convection in the flow. $\psi_{r}$ considers the influence of the difference of the retarded times between the two source positions and is defined by equation (24).

The product of coherence function times source interference function can have very large effects on the sound radiation of the source field and explains many features of the directivity of jet mixing noise. It will later be shown that this includes broadband shocknoise.

\section{Dipole noise}

The power-spectral density in equation (21) due to dipole noise is defined correspondingly.

$$
W_{p p d d}\left(x_{i}, f\right)=\frac{1}{\left(4 \pi a_{0}^{2}\right)^{2}} \underbrace{\int_{V} \frac{W_{d d s}}{r_{e}^{2} D_{f}^{4}} F_{d} \mathrm{~d} V\left(y_{i}\right)}_{\text {integral over source volume }}
$$

with

$$
F_{d}=\underbrace{\int_{V_{c}} \underbrace{\frac{r_{e} D_{f}^{2}}{r_{e c} D_{f c}^{2}}}_{\text {convect.ampl. }} \underbrace{\sqrt{\frac{W_{d d c}}{W_{d d s}}}}_{\text {source strength }} \underbrace{\gamma_{d}}_{\text {coherence }} \underbrace{\cos \left(\psi_{d}+\psi_{r}\right)}_{\text {source interference }} \mathrm{d} V_{c}\left(\eta_{i}\right)}_{\text {integral over coherence volume }},
$$

where $W_{d d s}$ and $W_{d d c}$ are the power-spectral densities of the dipole source function $Q_{d}$ (equation (20)) in the source volume elements $\mathrm{d} V\left(y_{i}\right)$ and $\mathrm{d} V_{c}\left(\eta_{i}\right)$, respectively. Note that the exponent of the Doppler factor $D_{f}$ is only 4 in equation (29) rather than 6 in equation (27). The consequence for flyover results is that the forward arc radiation will be more dominated by quadrupole noise than the rear arc and that the flight effects are larger for jets with constant density than for heated jets. 


\section{Geometric far field}

Eqs. (28) and (30) simplify if it is assumed that the observer position is located in the geometric far field of the coherence volume. The ratio $\left(r_{e} D_{f}^{2}\right) /\left(r_{e c} D_{f c}^{2}\right) \approx 1$. If it is further assumed that the source strength is almost constant within the coherence volume, we obtain

$$
\begin{gathered}
F_{q}=\underbrace{\int_{V_{c}} \underbrace{\gamma_{q}}_{\text {coherence source interference }} \underbrace{\cos \left(\psi_{q}+\psi_{r}\right)}_{F_{d}} \mathrm{~d} V_{c}\left(\eta_{i}\right)}_{\text {integral over coherence volume }}, \\
\underbrace{\int_{V_{c}} \underbrace{\gamma_{d}}_{\text {coherence source interference }} \underbrace{\cos \left(\psi_{d}+\psi_{r}\right)} \mathrm{d} V_{c}\left(\eta_{i}\right)}_{\text {integral over coherence volume }},
\end{gathered}
$$

The influence of the retarded time difference on the phase difference $\psi_{r}$ simplifies to

$$
\psi_{r}=2 \pi f \Delta t_{r}=2 \pi f \frac{\eta_{r_{e}}}{a_{0} D_{f}}=k \frac{\eta_{r e}}{D_{f}},
$$

where $\eta_{r_{e}}$ is the component in wave normal direction $\theta_{e}$ of the difference vector $\eta_{i}$ between the two source volume elements $\mathrm{d} V_{c}$ and $\mathrm{d} V$ as illustrated in figure 3 .

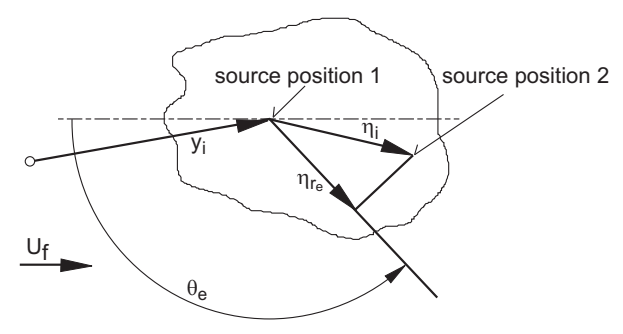

Figure 3. Definition of the vector $\eta_{i}$ which describes the difference between the two source volume elements and of its component $\eta_{r_{e}}$ in the wave normal direction.

\section{Normalization of equations}

With the nozzle diameter $D_{j}$ as reference length, $\Delta U_{j}=U_{j}-U_{f}$ as reference speed, $D_{j} / \Delta U_{j}$ as reference time the quadrupole source term $Q_{q}$ of equation (19) can be made dimensionless.

$$
Q_{q}\left(y_{i}, x_{i}, t\right)=Q_{q}^{\star}\left(y_{i}, x_{i}, t\right) \frac{\rho_{0} \Delta U_{j}^{2}}{\left(D_{j} / \Delta U_{j}\right)^{2}}=Q_{q}^{\star}\left(y_{i}, x_{i}, t\right) \frac{\rho_{0} \Delta U_{j}^{4}}{D_{j}^{2}},
$$

where the star indicates a nondimensional quantity, which will be assumed independent of $U_{j}, U_{f}, D_{j}, \rho_{j}$ in a first order approximation.

Michel \& Michalke ${ }^{9}$ considered the lengthening of the jet flow field in first order by introducing a jet stretching factor due to flight speed.

$$
\sigma=1+A U_{f} /\left(U_{j}-U_{f}\right), \quad A=1.5
$$

This yields

$$
\begin{aligned}
\mathrm{d} V & =\mathrm{d} V^{\star} \sigma D_{j}^{3}, \\
\mathrm{~d} V_{c} & =\mathrm{d} V_{c}^{\star} \sigma D_{j}^{3}, \\
F_{q} & =F_{q}^{\star} \sigma D_{j}^{3},
\end{aligned}
$$

where $V^{\star}$ and $V_{c}^{\star}$ are the normalized source and coherence volumes and $F_{q}^{\star}$ the interference integral of the static jet.

This stretching was theoretically verified by an instability theory of Michalke \& Hermann. ${ }^{17}$ They also showed that the normalized frequency of the instability waves in the shear layer is increased in flight, yielding

$$
f=f^{\star} \frac{\Delta U_{j}}{D_{j}} \sigma .
$$


where $f^{\star}$ is the normalized frequency of the static jet.

The cross-spectral density of the source term $Q_{q}$ in the source position $y_{i}$ is then given by

$$
W_{q q s}=\frac{\rho_{0}^{2} \Delta U_{j}^{7}}{\sigma D_{j}^{3}} W_{q q s}^{\star 2} .
$$

With $a_{0}^{2}=\gamma p_{0} / \rho_{0}(\gamma$ is the isentropic exponent) we finally obtain

$$
W_{p p q q}=\left(\frac{\gamma p_{0}}{4 \pi}\right)^{2}\left(\frac{D_{j}}{r_{e}}\right)^{2}\left(\frac{\Delta U_{j}}{a_{0}}\right)^{7} \frac{\sigma}{D_{f}^{6}} \int_{V} W_{q q s}^{\star} F_{q}^{\star} \mathrm{d} V^{\star}\left(y_{i}\right),
$$

where the nondimensional interference integral $F_{q}^{\star}$ is defined by

$$
F_{q}^{\star}=\int_{V_{c}^{\star}} \gamma_{q} \cos \left(\psi_{q}+\psi_{r}\right) \mathrm{d} V_{c}^{\star}\left(\eta_{i}\right)
$$

Integration over the whole frequency range $\left(0, f_{\max }^{\star}\right)$ yields for the quadrupole noise

$$
\overline{p_{q}^{2}}=\left(\frac{\gamma p_{0}}{4 \pi}\right)^{2}\left(\frac{D_{j}}{r_{e}}\right)^{2}\left(\frac{\Delta U_{j}}{a_{0}}\right)^{8} \frac{\sigma^{2}}{D_{f}^{6}} \int_{V} \int_{0}^{f_{\max }^{\star}} W_{q q s}^{\star} F_{q}^{\star} \mathrm{d} f^{\star} \mathrm{d} V^{\star}\left(y_{i}\right) .
$$

Corresponding results for the dipole noise are

$$
\begin{gathered}
W_{p p d d}=\left(\frac{\gamma p_{0}}{4 \pi}\right)^{2}\left(\frac{D_{j}}{r_{e}}\right)^{2}\left(\frac{\Delta U_{j}}{a_{0}}\right)^{5} \frac{\sigma}{D_{f}^{4}} \int_{V} W_{d d s}^{\star} F_{d}^{\star} \mathrm{d} V^{\star}\left(y_{i}\right), \\
F_{d}^{\star}=\int_{V_{c}^{\star}} \gamma_{d} \cos \left(\psi_{d}+\psi_{r}\right) \mathrm{d} V_{c}^{\star}\left(\eta_{i}\right), \\
\overline{p_{d}^{2}}=\left(\frac{\gamma p_{0}}{4 \pi}\right)^{2}\left(\frac{D_{j}}{r_{e}}\right)^{2}\left(\frac{\Delta U_{j}}{a_{0}}\right)^{6} \frac{\sigma^{2}}{D_{f}^{4}} \int_{V} \int_{0}^{f_{\max }^{\star}} W_{d d s}^{\star} F_{d}^{\star} \mathrm{d} f^{\star} \mathrm{d} V^{\star}\left(y_{i}\right) .
\end{gathered}
$$

\section{Interference as possible cause of directivity}

An inspection of equations (43) or (46) shows that a directivity pattern of jet noise can have various origins. The Doppler amplification due to the Doppler factor $D_{f}$ appears only in the flight condition. The directivity of a static jet can be caused by directivities of the power-spectral densities $W_{q q s}^{\star}$ or $W_{d d s}^{\star}$, and by a directivity of the interference functions $F_{q}^{\star}$ or $F_{d}^{\star}$. The latter depend according to equations (42) and (45) on $\gamma_{q}$ or $\gamma_{d}$, or on source interference effects caused by the terms $\cos \left(\psi_{q}+\psi_{r}\right)$ or $\cos \left(\psi_{d}+\psi_{r}\right)$. It will be shown, that many features of the directivity of jet noise can be explained with source interference effects.

It may be noted that the products $W_{q q s}^{\star} F_{q}^{\star}$ and $W_{d d s}^{\star} F_{d}^{\star}$ must be independent of the chosen source description. Other source descriptions were proposed for low Mach number flows by Ribner ${ }^{18}$ (pseudo-sound) and Powell ${ }^{19}$ (vorticity) or the modifications by Möhring ${ }^{20}$ and Obermeier. ${ }^{21}$ Although the sources are different as was pointed out by $\mathrm{Tam}^{22}$ the product of source spectral density times interference integral must yield the same result except for the influence of the simplifications. Different numerical values of $W_{q q s}$ must be compensated by the corresponding values of $F_{q}$.

\section{Analytical solutions with simple source model}

\section{A. Line model for jet}

Simplifications are required to make possible analytical solutions of the equations (31) and (32).

In a first step, the radial extension of the source volume is neglected. This is a drastic simplification since the far field of the jet becomes axisymmetric in contrast to experimental evidence of Maestrello. ${ }^{23}$ Nevertheless, we will see that major features of the directivity can be explained with this simple assumption. 
The line model allows to simplify the distance $\eta_{r_{e}}$ in equation (33) by

$$
\eta_{r_{e}}=\eta_{1} \cos \theta_{e}
$$

which yields

$$
\psi_{r}=k \eta_{1} \frac{\cos \theta_{e}}{D_{f}}
$$

The phase difference $\psi_{q}$ is caused by the convection of the turbulence in the shear layer of the jet. The phase difference between two source positions separated in the axial direction by $\eta_{1}$ may be approximated by a relation derived from the wave propagation of instability waves,

$$
\psi_{q}=2 \pi f \frac{\eta_{1}}{U_{p}\left(f, y_{i}, \eta_{i}\right)}=k \eta_{1} \frac{a_{0}}{U_{p}\left(f, y_{i}, \eta_{i}\right)} .
$$

$U_{p}\left(f, y_{i}, \eta_{i}\right)$ is the average phase velocity in the $\eta_{1}$-direction of the considered frequency component between positions $y_{i}$ and $y_{i}+\eta_{i}$. The phase angle $\psi=\psi_{q}+\psi_{r}$ in the interference function of equation (31) is then given by equations (48) and (49),

$$
\psi=\psi_{q}+\psi_{r}=k \eta_{1}\left(\frac{a_{0}}{U_{p}}+\frac{\cos \theta_{e}}{D_{f}}\right) .
$$

If we introduce a coherence length scale of the quadrupole sources

$$
L_{x q}=\int_{-\infty}^{\infty} \gamma_{q}\left(\eta_{1}\right) \mathrm{d} \eta_{1}
$$

and normalize the axial separation $\eta_{1}$ with $L_{x q}$, we obtain with $\xi=\eta_{1} / L_{x q}$

$$
\psi=k L_{x q}\left(\frac{a_{0}}{U_{p}}+\frac{\cos \theta_{e}}{D_{f}}\right) \xi .
$$

The nondimensional interference integral $F_{q}^{\star}$ according to equation (42) is then given by

$$
F_{q}^{\star}=L_{x q} / D_{j} \int_{-\infty}^{\infty} \gamma_{q} \cos \left(\psi_{q}+\psi_{r}\right) \mathrm{d} \xi
$$

The phase speed $U_{p}$ can be deduced from linear instability theory (Michalke \& Hermann $(1982)^{17}$ ), which yields frequency dependent values around

$$
U_{p}=U_{f}+0.7\left(U_{j}-U_{f}\right)
$$

\section{B. Mach wave radiation}

Maximum sound emission of a source volume element according to equations (31) or (32) is achieved for the smallest possible values of $\psi$ defined by equation (52). The phase $\psi$ vanishes according to equation (52) for an emission angle

$$
\cos \theta_{e M}=-\frac{a_{0}}{U_{p}-U_{f}}
$$

This condition can only occur if the relative phase speed $U_{p}-U_{f}$ of the jet is larger than the ambient speed of sound $a_{0}$,

$$
\frac{U_{p}-U_{f}}{a_{0}}>1
$$

The sound radiation into the angle defined by equation (55) is called Mach wave radiation. According to equation (54), the jet speed of a jet has to be at least 1.4 times the ambient speed of sound plus the flight speed for that to happen. For a given jet speed $U_{j}, U_{j}-U_{f}$ is smaller in flight than during static operation of the engine. This means that the Mach wave radiation angle $\theta_{e M}$ moves to the rear in flight. Mach wave radiation can even disappear in flight, which is the normal case for commercial aircraft in cruise. by

The power-spectral density of the sound pressure in the far field for the Mach wave radiation angle is then given

$$
W_{p p q q}\left(x_{i}, f\right)=\left(\frac{\gamma p_{0}}{4 \pi}\right)^{2}\left(\frac{D_{j}}{r_{e}}\right)^{2}\left(\frac{\Delta U_{j}}{a_{0}}\right)^{7} \frac{\sigma}{D_{f}^{6}} \frac{L_{x q}}{D_{j}} \int_{V} W_{q q s}^{\star} \mathrm{d} V^{\star}\left(y_{i}\right) .
$$


It is noteworthy that the contribution of a volume element $\mathrm{d} V^{\star}\left(y_{i}\right)$ in the source region to the frequency spectrum in the far field is identical to the frequency spectrum in the source region. The source spectrum is not altered by interference effects because $F_{q}^{\star}=1$ for Mach wave radiation.

Mach wave radiation is not restricted to small frequencies as claimed by Tam. ${ }^{22}$ The fact that higher frequencies are not observed in the rear arc may have two causes. Firstly, the effects of wave refraction which were experimentally verified by Atvars et al. ${ }^{24,25}$ are excluded in this simple model. They affect the spectral shape especially for high frequencies for angles close to the jet axis. Secondly, the interference in the radial direction is discarded in this simple model. The noise reduction through radial interference will necessarily be stronger when the wave length is small im comparison to the jet diameter. This can already be concluded from Michalke, ${ }^{5,7}$ who already discussed the noise emission of instability waves.

It may be noted that this result shows that Mach wave radiation can be described with the acoustic analogy in contrast to the claim of $\mathrm{Tam}^{22}$ and it exists for the quadrupole sources as well as for the dipole sources. The noise emission by instability waves is included in the solution for the geometric far field given by equation (27) for the quadrupole sources and equation (29) for the dipole sources.

\section{Coherence models}

Three different coherence models shall be investigated. For each of them a closed form solution exists for the interference integral

$$
F_{r}=F_{q}^{\star} D_{j} / L_{x q}=\int_{-\infty}^{\infty} \gamma_{q}(\xi) \cos \left(2 \pi \frac{f L_{x q}}{U_{p}}\left[1+\frac{U_{p}}{a_{0}} \frac{\cos \theta_{e}}{D_{f}}\right] \xi\right) \mathrm{d} \xi .
$$

Model 1 describes a coherence between two axially displaced positions in a nozzle-fixed coordinate system that decays exponentially with the normalized separation distance $\xi=\eta_{1} / L_{x q}$ according to

$$
\gamma=\exp (-2|\xi|)
$$

The length scale $L_{x q}$ is a function of frequency in this frequency dependent description. ${ }^{\text {a }}$

A second shape for the coherence function is given by

$$
\gamma=\frac{1}{1+\pi^{2} \xi^{2}} .
$$

The coherence of turbulent fluctuations in a frame moving with the flow is often modeled by the Gauss function. If we assume such a behavior in the nozzle-fixed coordinate system we have

$$
\gamma=\exp \left(-\pi \xi^{2}\right)
$$

The three coherence models are compared in figure 4. The coherence functions of models 2 and 3 have a gradient zero for $\xi=0$. Model 3 has the widest distribution close to $\xi=0$ but the most rapid decay for large separations $\xi$.

\section{Interference integrals}

The interference effect is the result of the interference integral of equation (58). Using the coherence model 1 of equation (59) we obtain the analytical solution

$$
F_{r 1}=\frac{1}{1+\left[\pi\left(f L_{x q} / U_{p}\right)\left(1+\left(U_{p} / a_{0}\right) \cos \theta_{e} / D_{f}\right)\right]^{2}} .
$$

The integral based on coherence model 2 of equation (60) is

$$
F_{r 2}=\exp \left[-2 \frac{f L_{x q}}{U_{p}}\left|\left(1+\frac{U_{p}}{a_{0}} \frac{\cos \theta_{e}}{D_{f}}\right)\right|\right]
$$

and the interference integral with coherence model 3 of equation (61) is

$$
F_{r 3}=\exp \left[-\pi\left(\frac{f L_{x q}}{U_{p}}\left(1+\frac{U_{p}}{a_{0}} \frac{\cos \theta_{e}}{D_{f}}\right)\right)^{2}\right]
$$

\footnotetext{
${ }^{a}$ The turbulence length scale in numerical computations is defined differently in a moving frame of reference and as an integral value over the whole frequency range.
} 


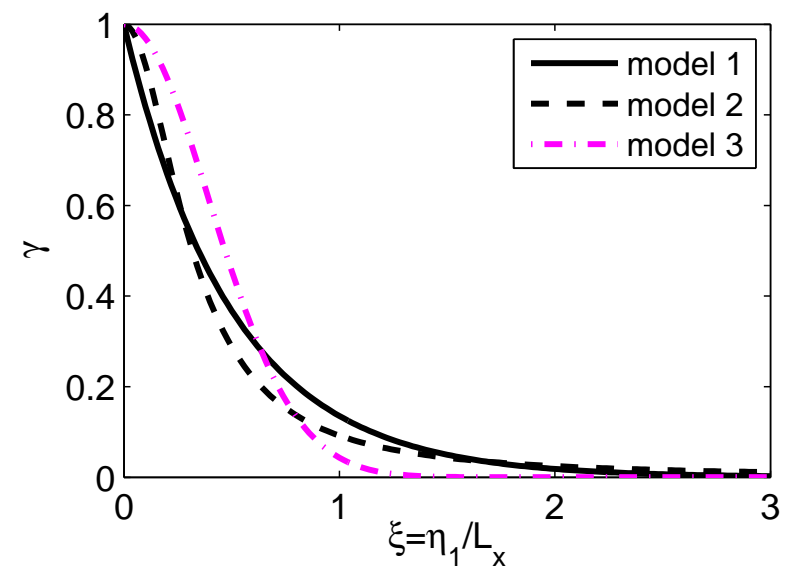

Figure 4. Comparison of the three investigated coherence models.

\section{E. Influence of axial length scale}

The influence of the Strouhal number $f L_{x q} / U_{p}$, which can be considered a normalized length scale, is studied first. The result for coherence model 1 and an acoustic Mach number of the convected turbulence $U_{p} / a_{0}=1.2$ and for the flight Mach number $M_{f}=0$ is shown in figure 5(a) as a function of emission angle $\theta_{e}$.

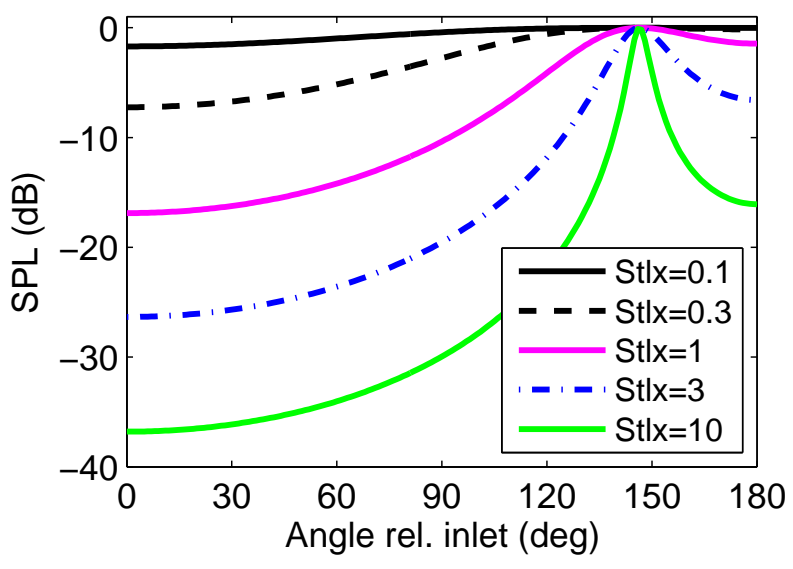

(a) $U_{p} / A_{0}=1.2$

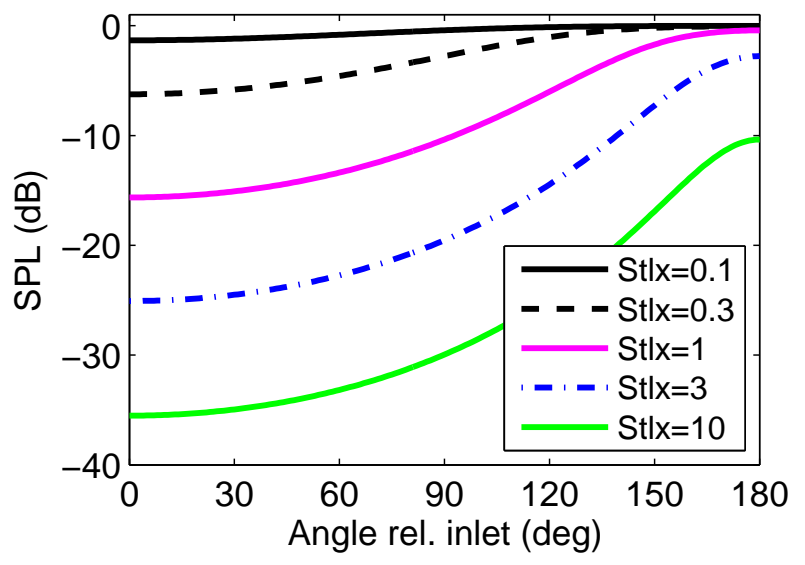

(b) $U_{p} / A_{0}=0.9$

Figure 5. Interference integral for various length scales $f L_{x q} / U_{p}$ and two phase Mach numbers $U_{p} / a_{0}$ with coherence model 1. The maximum at $146^{\circ}$ in the left figure is due to Mach wave radiation.

It can be seen that the convective amplification in the rear arc depends strongly on the length scale $f L_{x q} / U_{p}$ of the source distribution in the turbulent flow field. The curves reach a value of $0 \mathrm{~dB}$ for the Mach wave radiation angle of $\theta_{e M}=146^{\circ}$. The directivity is reduced due to interference for all other angles, the effect increases with the length scale. The result for one value for $f L_{x q} / U_{p}$ is valid for all frequencies if the product $f L_{x q}$ remains constant for a constant phase speed $U_{p}$. This is the case if the length scale is proportional to the wave length of the generated sound.

The results for a subsonic phase speed $U_{p} / a_{0}=0.9$ are shown in figure $5(\mathrm{~b})$. The directivities peak at $180^{\circ}$ because the refraction dimple ${ }^{24,25}$ in the rear arc due to wave refraction is not considered by this simple model.

The next two figures 6(a) and 6(b) describe the same results for the coherence model 2. It can be seen that the level reduction in the forward arc due to interference is much larger for coherence model 2 than for model 1.

The next two figures 7(a) and 7(b) describe the same results for the coherence model 3. The level reduction in the forward arc due to interference is even larger for coherence model 3. The reduction is unrealistically large. Therefore, model 3 will not be studied further because it is not applicable for the fixed frame of reference used here.

It can be concluded that convective amplification requires large coherence length scales in the streamwise direction. The experimentally observed convective amplifications are compatible with $f L_{x} / U_{p} \approx 1$. For a Strouhal number 


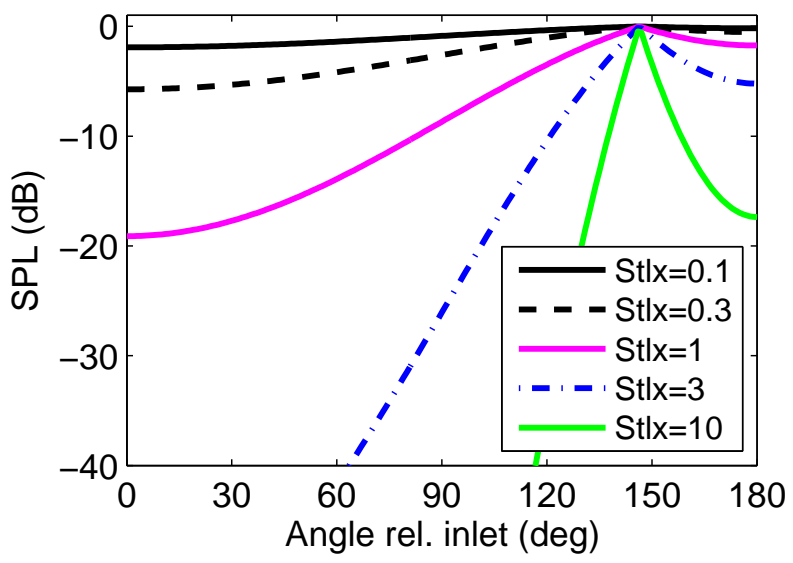

(a) $U_{p} / A_{0}=1.2$

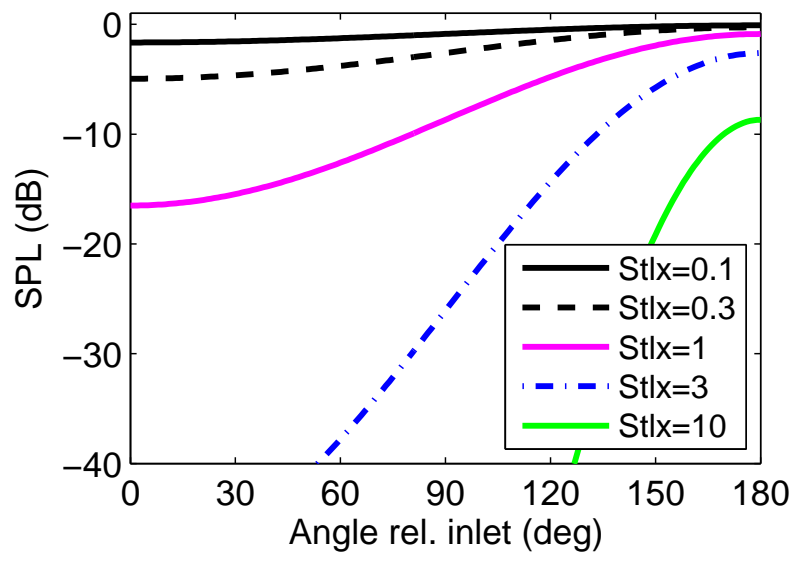

(b) $U_{p} / A_{0}=0.9$

Figure 6. Interference integral for various length scales $f L_{x q} / U_{p}$ and two phase Mach numbers $U_{p} / a_{0}$ with coherence model 2. The maximum at $146^{\circ}$ in the left figure is due to Mach wave radiation.

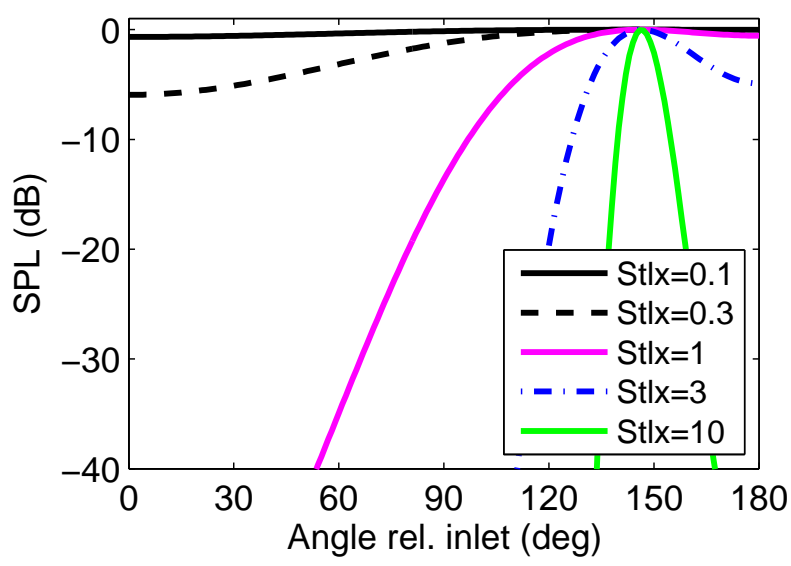

(a) $U_{p} / A_{0}=1.2$

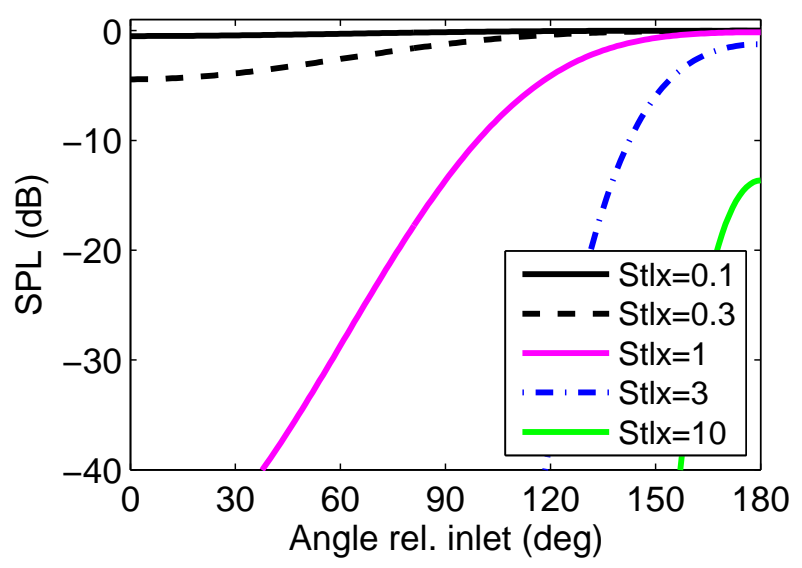

(b) $U_{p} / A_{0}=0.9$

Figure 7. Interference integral for various length scales $f L_{x q} / U_{p}$ and two phase Mach numbers $U_{p} / a_{0}$ with coherence model 3. The maximum at $146^{\circ}$ in the left figure is due to Mach wave radiation.

$f D_{j} / U_{j}=1.0$ (peak Strouhal number of one-third octave band spectra) and $U_{p} / U_{j}=0.7$ the length scale is $L_{x} \approx$ $0.7 D_{j}$.

\section{F. Influence of axial phase speed}

The influence of the acoustic phase Mach number $M a_{p}=U_{p} / a_{0}$ is studied next for a length scale $f L_{x q} / U_{p}=1$. The results for models 1 and 2 are shown in figures 8(a) and 8(b). The resulting directivity shapes are as expected, since the convective amplification in the rear arc increases with $U_{p} / a_{0}$. The spread between the curves in the forward arc is larger for model 2.

\section{G. Influence of flight Mach number}

The influence of the flight Mach number is studied now for the two coherence models. The phase speed $U_{p} / a_{0}=1.2$ and the length scale $f L_{x q} / U_{p}=1$. The results are shown in figures 9(a) and 9(b). The Doppler amplification $D_{f}^{-6}$ for the quadrupole sources (see equation (41)) is considered. The influences of the changed relative jet speed $U_{j}-U_{f}$, of the factor $L_{x} / D_{j}$ in equation (53), and of a possible jet stretching in flight are not included. Therefore, the directivity values remain uninfluenced by $M a_{f}$ at $\theta_{e}=90^{\circ}$. Note that the chosen coherence model has a large influence on the sound radiation into the forward arc. 


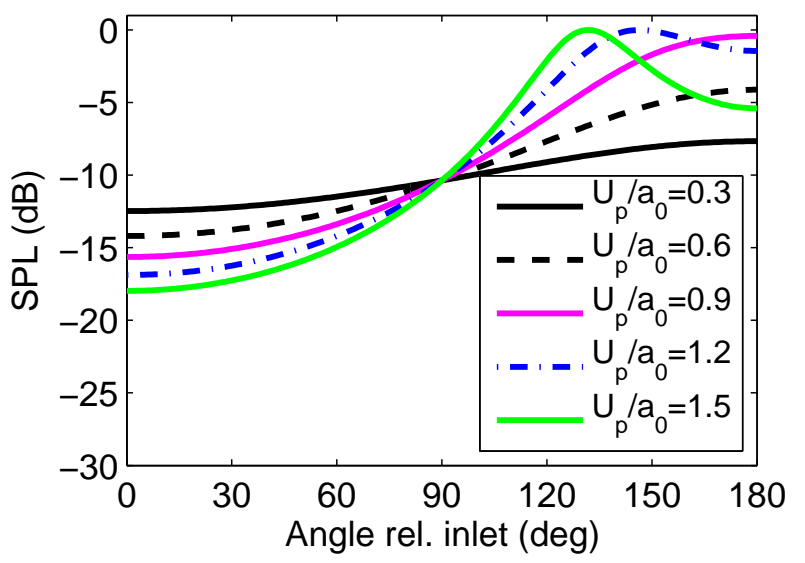

(a) Model 1

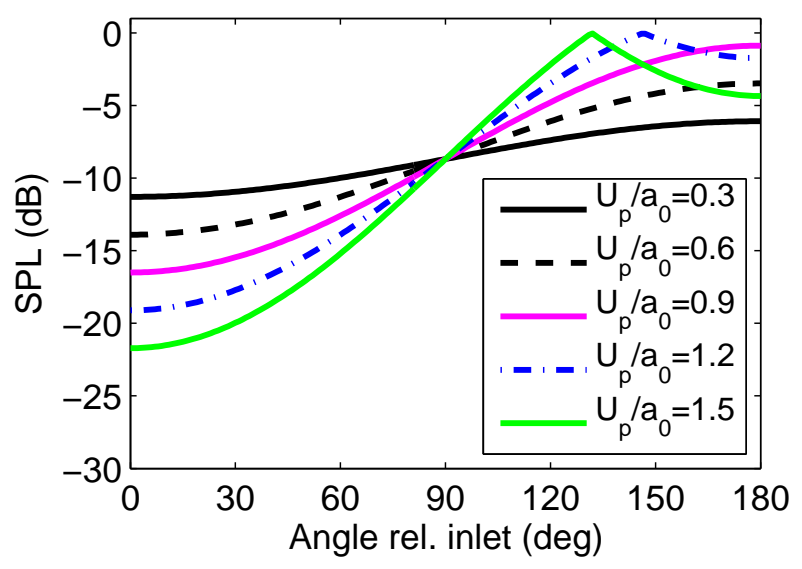

(b) Model 2

Figure 8. Interference integral for various phase Mach numbers $U_{p} / a_{0}$ and $f L_{x q} / U_{p}=1$.

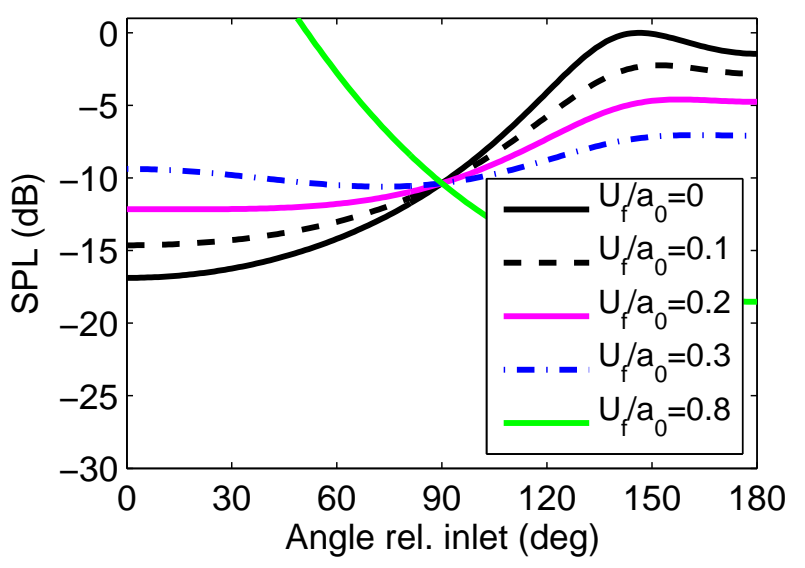

(a) Model 1

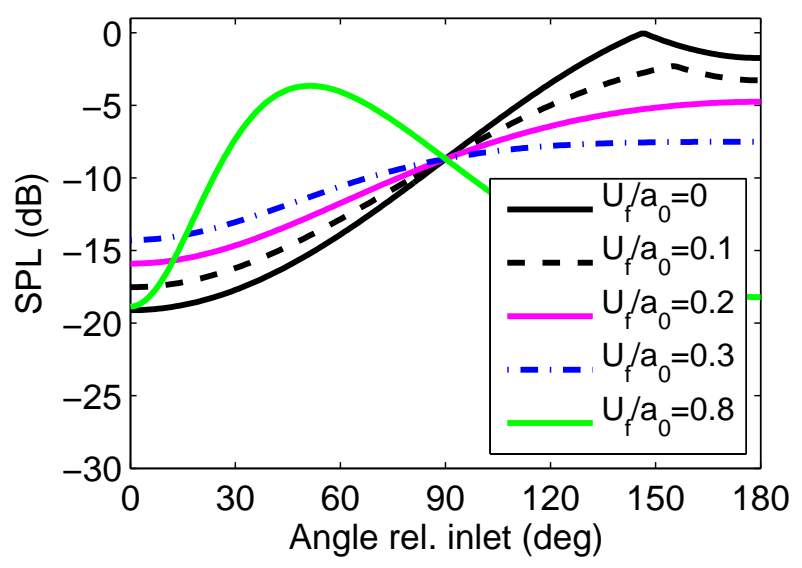

(b) Model 2

Figure 9. Interference integral for various flight Mach numbers $U_{f} / a_{0}$ with $f L_{x q} / U_{p}=1$ and $U_{p} / a_{0}=1.2$. Doppler amplification $D_{f}^{-6}$ for quadrupole sources included. The radiation into the forward arc, escpecially at cruise Mach numbers depends strongly on the chosen coherence model. The actual behavior of a jet in cruise cannot be derived, but a high noise emission into the forward arc cannot surprise.

The forward arc amplification becomes very strong for flight Mach numbers during cruise but depends considerably on the chosen coherence model. The actual behavior of a jet in cruise cannot be predicted with this simple model but a high noise emission into the forward arc cannot surprise. Since the jet is stretched substantially at cruise Mach numbers, the low frequency jet sources are located much farther downstream than in a static condition. The consequence is that the cabin of an aircraft may be located in the main beam of jet mixing noise during cruise.

The results for dipole sources, where the Doppler amplification is $D_{f}^{-4}$ are shown in figures 10 (a) and 10 (b). The forward arc amplification is slightly smaller.

\section{H. Influence of acoustic jet Mach number}

The influence of the acoustic jet Mach number $U_{j} / a_{0}$ is shown in figure 11(a) for the coherence model 1 and in figure 11(b) for coherence model 2. for three emission angles $\theta_{e}=60^{\circ}, 90^{\circ}, 120^{\circ}$. The flight Mach number $M_{f}=0$. Lighthill's result of $\overline{p^{2}} \propto\left(U_{j} / a_{0}\right)^{8}$ for an emission angle of $\theta_{e}=90^{\circ}$ is recovered. 


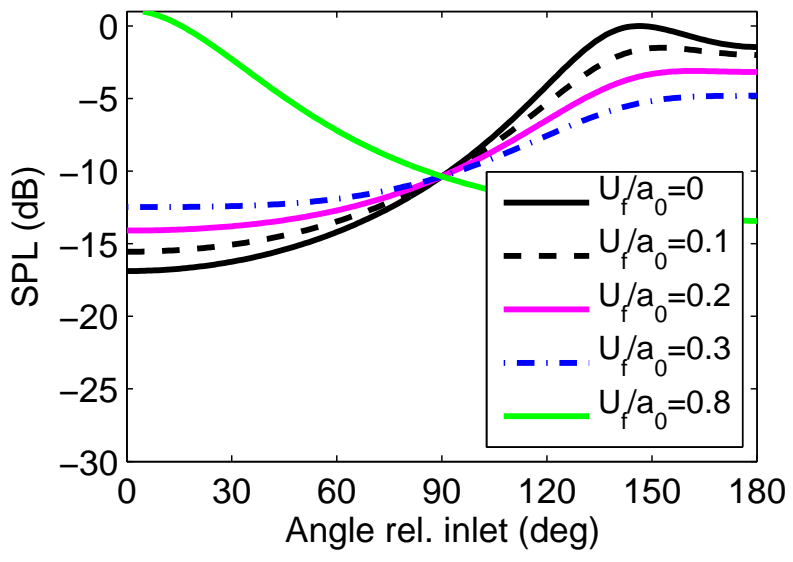

(a) Model 1

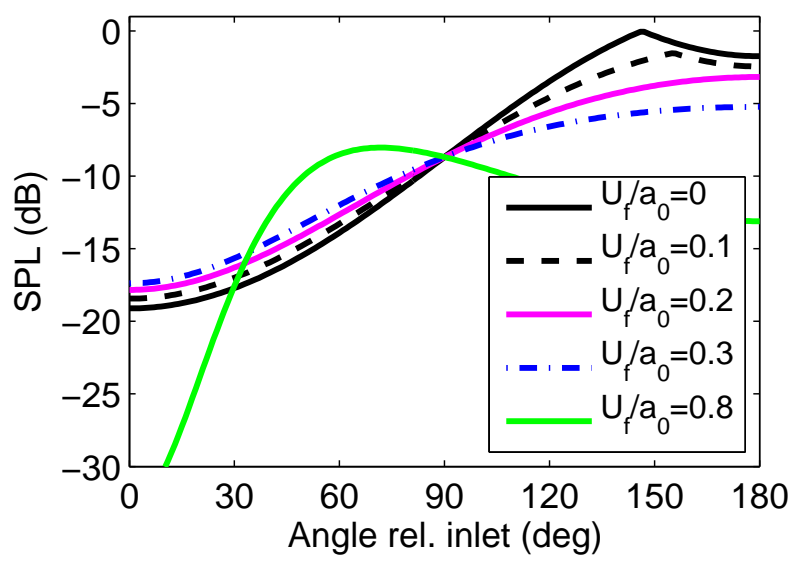

(b) Model 2

Figure 10. Interference integral for various flight Mach numbers $U_{f} / a_{0}$ with $f L_{x q} / U_{p}=1$ and $U_{p} / a_{0}=1.2$. Doppler amplification $D_{f}^{-4}$ for quadrupole sources included. The radiation into the forward arc, escpecially at cruise Mach numbers depends strongly on the chosen coherence model.

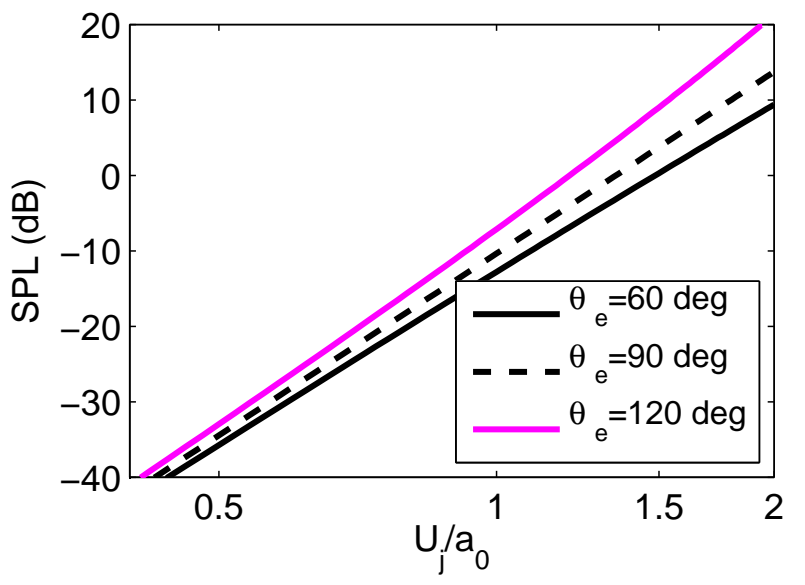

(a) Model 1

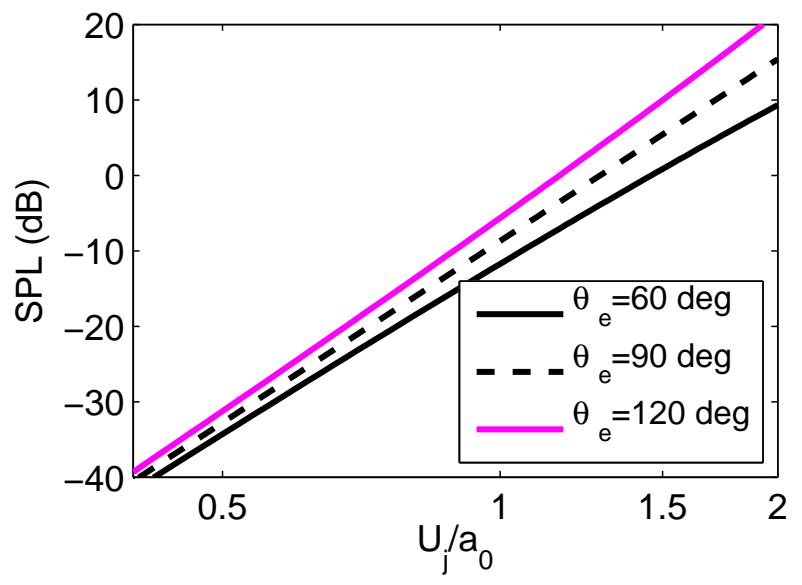

(b) Model 2

Figure 11. Influence of acoustic jet Mach number $U_{j} / a_{0}$ and emission angle $\theta_{e}$ on the sound radiation in a one-third octave band for $f L_{x q} / U_{p}=1$ and a flight Mach number of $U_{f} / a_{0}=0$. The results for $90^{\circ}$ agree with Lighthill's result of $\overline{p^{2}} \propto\left(U_{j} / a_{0}\right)^{8}$. 


\section{Conclusions}

The sound radiation of a free jet is studied in the frequency domain. An integral for the power-spectral density is derived, which includes the quadrupole sources for jets with constant mean density and the additional dipole sources of jets with nonuniform density. In contrast to the theories of Lighthill, ${ }^{1,2}$ Ffowcs Williams, ${ }^{3}$ and many others, the analysis is performed in a coordinate system fixed on the nozzle of the jet, where the source terms satisfy the mathematically important condition of stationary randomness and the limits of the source integral are stationary. Turbulence convection is considered through a phase angle of the cross-spectral density of the sources. The influence of source interference is expressed in terms of an interference integral which describes the sound radiation of one source volume element including the interference effects with its neighborhood.

In order to achieve analytic solutions for this integral, the radial extension of the jet is neglected and simple models are introduced for the decay of the coherence with increasing axial separation of the source positions. The interference effects reduce the sound emission for all angles except for the Mach wave radiation angle. Some main features of jet mixing noise can be explained with this simple model, the convective amplification, the Mach wave radiation and the independence of frequency with emission angle.

However, the model is admittedly crude. The neglect of the radial extension of the jet may be an acceptable approximation for long wave lengths, but it is certainly not valid for short wave lengths. The radial extension can be approximately considered when the source region is modeled by a cylindrical source distribution. This allows to decompose the cross-spectral density into azimuthal components according to Michalke ${ }^{6}$ and a separate treatment of each component. The effect of radial interference should be largest in the case of Mach wave radiation where the low frequencies suffer no attenuation due to interference, while the attenuation due to radial interference is not negligible for higher frequencies. Also neglected are the effects of wave refraction which affect the radiation into the rear arc.

The results are

- The frequency in the far field is independent of source motion.

- The spectral shape is determined by interference effects, which are a function of frequency.

- The convective amplification in the rear arc depends on the axial length scale of the turbulence.

- Length scales in the order of one jet diameter are required to explain the measured convective amplification for the peak frequency of jet mixing noise.

- The coherence needs to decay rather rapidly in the axial direction for small separation distances in order to explain the experimentally observed directivities.

- The Gauss function is not a suitable assumption to describe the axial decay of coherence between two source positions in a nozzle fixed coordinate system.

- The Mach wave radiation for supersonic convection speeds is correctly described.

- The separation between jet noise from large scale turbulence and small scale turbulence found in the literature might be explainable as jet noise radiation with small source interference and large source interference effects.

- The flight speed yields a convective amplification of jet noise in the forward arc. The actual amplification depends strongly on the chosen coherence model.

- Jet noise should peak in the forward arc for cruise Mach numbers according to the results of the interference integral.

- The integral describing the noise emission of one volume element must be independent of the source definition, i.e., it should be applicable to the pseudo sound and the vortex sound source terms for low Mach number jets.

- The result for the noise emission of one volume element might be suitable for post-processing numerical simulation results.

- The result for the noise emission of one volume element might also help develop improved source localization procedures for the analysis of jet mixing noise on engine test beds.

\section{Acknowledgments}

The work was co-financed by the German Federal Ministry of Economics and Technology within the research project LEXMOS (Quiet nozzle systems and sound source localization). 


\section{References}

${ }^{1}$ Lighthill, M. J., "On Sound Generated Aerodynamically. I. General Theory,” Proc. Roy. Soc. London, Vol. A 211, 1952, pp. 564-587.

${ }^{2}$ Lighthill, M. J., "On Sound Generated Aerodynamically. II. Turbulence as a Source of Sound," Proc. Roy. Soc. London, Vol. A 222, 1954, pp. 1-32.

${ }^{3}$ Ffowcs Williams, J. E., "The Noise from Turbulence Convected at High Speed," Phil. Trans. Roy. Soc., Vol. A 225, 1963, pp. $469-503$.

${ }^{4}$ Ribner, H. S., "New Theory of Jet-Noise Generation, Directionality, and Spectra," J. Acoust. Soc. Am., Vol. 31, 1959, pp. $245-246$.

${ }^{5}$ Michalke, A., "A Wave Model for Sound Generation in Circular Jets," Deutsche Luft- und Raumfahrt, DLR FB 70-57, 1970.

${ }^{6}$ Michalke, A., "An Expansion Scheme for the Noise From Circular Jets," Z. Flugwiss., Vol. 20, 1972, pp. 229-237.

${ }^{7}$ Michalke, A., "On the Effect of Spatial Source Coherence on the Radiation of Jet Noise," J. Sound Vib., Vol. 55, 1977, pp. 377-394.

${ }^{8}$ Michalke, A., "Some Remarks on Source Coherence Affecting Jet Noise," J. Sound Vib., Vol. 87, 1983, pp. 1-17.

${ }^{9}$ Michalke, A. and Michel, U., "Prediction of Jet-Noise in Flight from Static Tests," J. Sound Vib., Vol. 67, 1979, pp. 341-367.

${ }^{10}$ Michalke, A. and Michel, U., "Importance of Jet Temperature on the Prediction of Jet-Noise in Flight," Mechanics of Sound Generation in Flows, edited by E.-A. Müller, Springer-Verlag Berlin Heidelberg, 1979, pp. 256-263, Proceedings of the IUTAM/ICA/AIAA-Symposium, Göttingen, August 28-31, 1979.

${ }^{11}$ Michel, U. and Michalke, A., "Prediction of Flyover Jet Noise Spectra," AIAA Paper 81-2025, 1981, AIAA 7th Aeroacoustics Conference.

${ }^{12}$ Michel, U. and Michalke, A., "Prediction of Flyover Jet Noise Spectra From Static Tests," NASA Technical Memorandum 83219, 1981.

${ }^{13}$ Michel, U., "Broadband Shock Noise: Theory Vis-A-Vis Experimental Results," First Joint CEAS/AIAA Aeroacoustics Conference (16th AIAA Aeroacoustics Conference), 1995, pp. 545 - 554, CEAS/AIAA Paper 95-071, DGLR-Bericht 95-01.

${ }^{14}$ Tam, C. K. W. and Auriault, L., "Jet Mixing Noise from Fine-Scale Turbulence," AIAA J., Vol. 37, 1999, pp. 145-153.

${ }^{15}$ Ffowcs Williams, J. E., "Hydrodynamic Noise," Annual Review of Fluid Mechanics, Vol. 1, 1969, pp. 197-222.

${ }^{16}$ Morfey, C. L., "Amplification of Aerodynamic Noise by Convected Flow Inhomogeneities," J. Sound Vib., Vol. 31, 1973, pp. $391-397$. 359.

${ }^{17}$ Michalke, A. and Hermann, C., "On the Inviscid Instability of a Circular Jet With External Flow," J. Fluid Mech., Vol. 114, 1982, pp. 343-

${ }^{18}$ Ribner, H. S., "Aerodynamic Sound from Fluid Dilatations, A Theory of the Sound from Jets and other Flows," Report 86, Institute of Aerophysics, University of Toronto, 1962.

${ }^{19}$ Powell, A., "Theory of Vortex Sound," J. Acoust. Soc. Am., Vol. 36, 1964, pp. 177-195.

${ }^{20}$ Möhring, W., "On Vortex Sound at Low Mach Number," J. Fluid Mech., Vol. 85, 1978, pp. 685-691.

${ }^{21}$ Obermeier, F., "On a New Representation of Aeroacoustic Source Distribution. I: General Theory. II: Two-dimensional Model Flows." Acustica, Vol. 42, 1979, pp. 56.

${ }^{22}$ Tam, C. K. W., "Computational Aeroacoustics Examples Showing the Failure of the Acoustic Analogy Theory to Identify the Correct Noise Sources," J. Comp. Acoustics, Vol. 10, 2002, pp. 387-405.

${ }^{23}$ Maestrello, L., "Statistical Properties of the Sound and Source Fields of an Axisymmetric Jet," AIAA Paper 77-1267, 1977, AIAA 4th Aeroacoustics Conference.

${ }^{24}$ Atvars, J., Schubert, L. K., and Ribner, H. S., "Refraction of Sound from a Point Source Placed in an Air Jet," J. Acoust. Soc. Am., Vol. 37, 1965 , pp. 168.

${ }^{25}$ Atvars, J., Schubert, L. K., Grande, E., and Ribner, H. S., "Refraction of Sound by Jet Flow or Jet Temperature," TN 109 (1965); NASA CR-494 (1966), University of Toronto, Institute for Aerospace Studies, 1965. 